\title{
Research Paper: The Relationship Between Trunk Muscle Endurance and Three-dimensional Knee Kinematics During Jump-Landing Among Military Personnel
}

\author{
Mohammad Fallah Mohammadi $^{1 *}$ (D) Vahid Sobhani $^{1}$ (D)
}

1. Exercise Physiology Research Center, Lifestyle Institute, Baqiyatallah University of Medical Sciences, Tehran, Iran.

\begin{tabular}{|l|l}
\hline $\begin{array}{l}\text { Use yourdevice to scan } \\
\text { and read the artice online }\end{array}$ \\
Knee Kinematics During Jump-Landing Among Military Personnel. Journal of Modern Rehabilitation. 2019; 13(3):161-170. \\
http://dx.doi.org/10.32598/JMR.13.3.161 \\
dol'http://dx.doi.org/10.32598/JMR.13.3.161
\end{tabular}

Article info:

Received: 13 Dec 2018

Accepted: 25 Mar 2019

Available Online: 01 Jul 2019

\section{Keywords:}

Core muscle endurance, Jumplanding, McGill protocol, Knee joint alignment

\section{ABSTRACT}

Introduction: Musculoskeletal injuries are prevalent among active individuals, such as military personnel, which may lead to high health costs. The injuries, specifically in lower extremity joints, are mostly taking place during movements such as jumping. This study investigates the correlation between trunk muscle endurance and three-dimensional knee kinematics during jump-landing among military personnel.

Materials and Methods: Thirty commandoes (Mean \pm SD age: $29.45 \pm 4.76)$ participated in the study. The researchers performed McGill standardized testing battery (core muscle endurance tests). Our subjects underwent jump-landing maneuver and 3D motion analysis of knee joint kinematics. Then the investigators analyzed the obtained data using the Pearson correlation test $(\mathrm{P}<0.05)$. The main outcome measures were trunk muscles' endurance and knee joint angles of flexion-extension, abduction-adduction, and internal rotation-external rotation estimated using MATLAB scripts for three trials during peak angle of knee flexion just before the rebound. We also calculated the average value for the aforementioned motions from initial contact to the take-off phase.

Results: The Pearson correlation test indicated a statistically significant correlation between the peak and average value of knee flexion angle and right $\left(\mathrm{r}_{\text {peak }}=0.511, \mathrm{r}_{\text {average }}=0.505\right.$ / $\left.\mathrm{P}_{\text {peak }}=0.021, \mathrm{P}_{\text {average }}=0.023\right)$ and left $\left(\mathrm{r}_{\text {peak }}=0.486, \mathrm{r}_{\text {average }}=0.445 / \mathrm{P}_{\text {peak }}=0.030, \mathrm{P}_{\text {average }}=0.049\right)$ side bridge tests. Moreover, we observed a significant negative correlation between average knee internal rotation and trunk flexors' endurance test value $(\mathrm{r}=-0.496, \mathrm{P}=0.026)$.

Discussion: The trunk muscles provide knee stability in dynamic tasks. Particularly, strengthening abdominal and lateral muscles of the trunk high shown to decrease knee rotation and flexion, respectively, during landing from a jump. Consequently, the authorities should emphasize this fact while designing conditioning or rehabilitation programs.

* Corresponding Author: 


\section{Introduction}

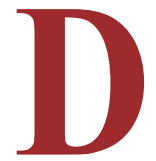

espite its unclear nature, core stability is quite popular; in other words, its definition depends on subjective viewpoint: ranging from hip and trunk muscle strength, trunk muscle endurance, maintenance of a particular pelvic inclination or vertebral alignment, and ligamentous laxity of the vertebral column [1]. The activation of core muscles is essential to stabilize the spine, allow endurance loads, and produce gross movement of the entire core structure. Therefore, several areas of core stability should be considered in this regard. Researchers indicate that abdominal muscle should activate before the prime mover to provide a foundation for limb movement [2]. They also note that the deepest abdominal muscle, the transversus abdominis, was invariably the first muscle automatically activated to prepare body movement, followed by the multifidus [1]. Further, Bouisset (1991) suggested that motor activity in the form of postural support must occur before the initiation of extremity movements [3]

Core stability prevents injury and increases performance enhancement. Researchers suggest-although based on limited studies-that adding core stabilization exercise to injury prevention programs, especially for lower extremities, can decrease injury rate [4]. Concerning dynamic stability, the endurance of the core musculature has often been thought of as a possible predictor for injury. It shows that core stability and proprioception has been an indicator of lower extremity injury. Among these, non-contact Anterior Cruciate Ligament (ACL) injuries are prevalent in sports and usually occur during dynamic activities of deceleration, such as jumping or changing direction [5].

During such events, because of the internal rotation of the hip, adduction of the femur, and external rotation of the tibia, the knee is often subjected to excessive frontal plane motion [6]. Mechanics of the trunk, hip, and knee are critical factors in noncontact ACL injury etiology. They also provide a link between ACL injury and proximal lower extremity [5, 7]. Several works suggest that muscles, such as the transversus abdominis, obliques, and multifidi, are useful in spinal stabilization. Therefore, dysfunction of the muscles is associated with dangerous movement patterns [6].

As noted, landing and jumping are frequent in the military. Often military personnel performs ground movements, or they are required to jump and land in a position demanding quick responses. The landing strategy has an inherent risk of a knee injury. Sell et al. reported that ankle and knee injuries are prevalent in military personnel that account for $10.9 \%$ to $15.1 \%$ and $10.2 \%$ to $12.0 \%$, respectively, of all musculoskeletal injuries in this population [5]

Zazulak et al. demonstrated that proprioceptive deficits in the body's core might contribute to decreased active neuromuscular control of the lower extremity, leading to the valgus orientation of the knee [8]. For efficient movement, segments must move in a proximal to the distal manner through the kinetic chain [9]. When muscles perform synergistically from proximal to distal, the result is postural control that allows for coordinated movements [10]. The coordinated movements of the lumbopelvic and hip complex provide the ability to maintain trunk positioning or postural awareness after static and dynamic muscular contractions [11].

Unfortunately, the role of core muscles in lower extremity kinematics during a functional task is not fully understood [6]. Thus, this study examines the relationship between the core muscles of the trunk and knee kinematics during two-legged jump-landing among army personnel.

\section{Materials and Methods}

Thirty active military personnel participated in this correlational cross-sectional study with consent. The

Table 1. Subject demographics

\begin{tabular}{ccc}
\hline Variables & Mean \pm SD & Range \\
\hline Height $(\mathrm{m})$ & $178.5 \pm 7.37$ & $160-188$ \\
Weight $(\mathrm{kg})$ & $79.20 \pm 11.08$ & $60-97$ \\
$\mathrm{BMI}, \mathrm{kg} / \mathrm{m}^{2}$ & $24.77 \pm 2.40$ & $20.8-30.6$ \\
\hline Age, $\mathrm{y}$ & $29.45 \pm 4.76$ & $20-37$ \\
\hline
\end{tabular}

BMI: Body Mass Index 
researchers determined the number of subjects using G-Power sample size calculation software (with an effect size of 0.50 , power of 0.80 , and alpha of 0.05 ). The subjects were older than 18 years and could perform standard physical training with no conditions that may affect jump-landing performance. The presence of low back pain and the inability to perform the core stability tests were the other criteria. Table 1 lists demographic characteristics for all participants.

\section{Core endurance}

The researches assessed the core endurance by recording the time (using chronometer). Each participant can maintain the positions of side bridge, prone plank, trunk flexor, and extensor endurance tests considering McGill and colleagues protocols. The reliability coefficients of the tests were greater than 0.97 [12]. The side bridge test was performed while the feet were placed one on top of the other, the right upper arm was perpendicular to the ground with the elbow resting on the mat, and the left arm was placed over the chest, with the left hand on the right shoulder. If any other part of the subject's body touched the floor, the examiner would stop the time (Figure 1a). The side bridge test is "suggested to challenge optimally quadratus lumborum and the muscles of the anterolateral (core) wall"' [13].

For the prone plank, the subject lays prone off the floor. The test starts when the subject lays in a horizontal position, with arms crossed over the chest. If the subject drifted from the horizontal level, the examiner woud

A

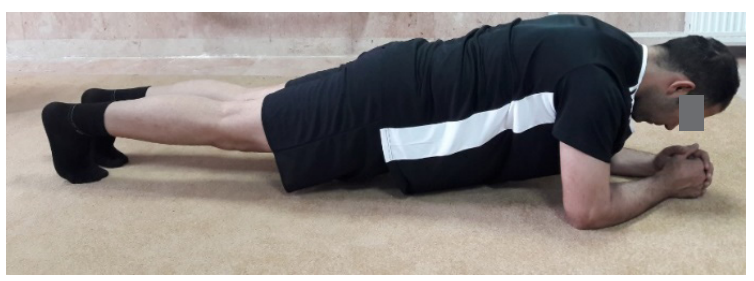

Side bridge test

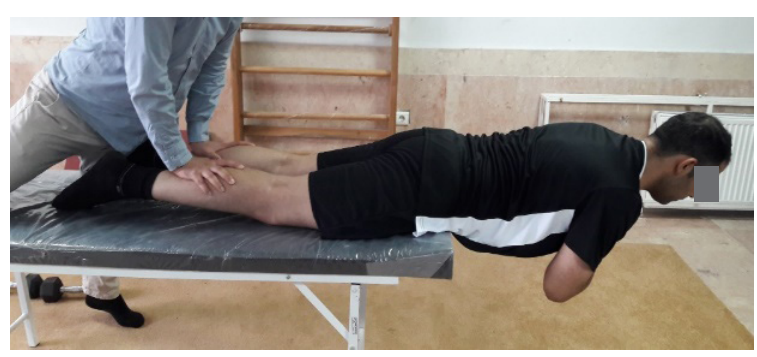

Trunk extensor test

Figure 1. Mcgill protocol for measuring core muscle endurance stop the time (Figure $1 \mathrm{~b}$ ). The trunk extensor endurance test provides a "global measure of back extension endurance capacity" [14] (Figure $1 \mathrm{c}$ ). The flexor endurance test was performed while the feet were placed flat on the table and fixed using two dumbbells, and the knees and hips were at approximately $90^{\circ}$ of flexion. The subject started with his back against a wooden wedge $60^{\circ}$ from the horizontal. Time began when the wedge was moved $10 \mathrm{~cm}$ back from the subject and ended when the subject's back touched the wedge [13] (Figure $1 \mathrm{~d}$ ).

\section{Jump-landing test}

The jump-landing task consists of a jump from a 30 $\mathrm{cm}$ box to a distance of $50 \%$ of the subject's height away from the box and immediately rebounded for a maximal vertical jump on landing. Subjects were not provided any instructions on their landing technique unless they perform the task incorrectly. A successful jump has five characteristics: jumping off of both feet from the box; jumping forward, but not vertically, to reach the floor; landing with the entire foot of the dominant lower extremity on the floor; landing with the whole foot of the non-dominant lower extremity off the floor, and completing the task in a fluid motion [15]. If the test lacked one of the features, the subjects would repeat the drill.

\section{Biomechanical analysis}

The researchers analyzed the kinematics by using the 3D motion analysis during the jump landing test. They also followed a lower-body point cluster technique

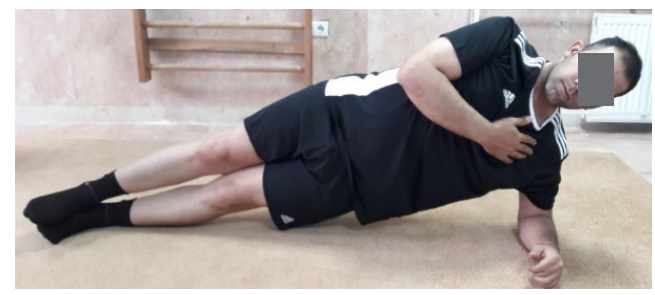

Prone plank test

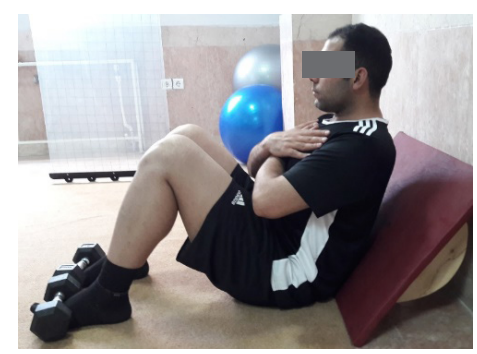

Trunk flexor test 


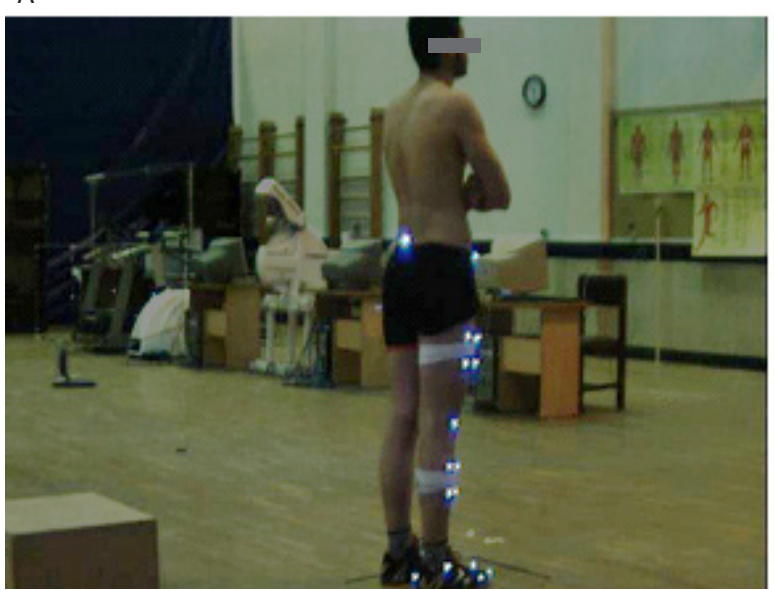

Sagittal view of the marker set
B

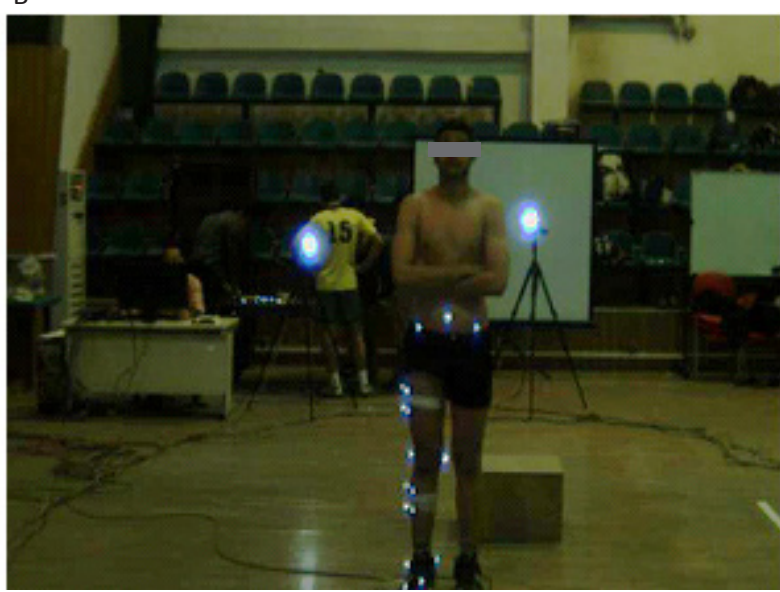

Frontal view of the marker set

Figure 2. Marker set in a static position (anatomical)

marker set [Qualysis Inc., Savebalden, Sweden] to estimate lower extremity kinematics (Figure 2, Figure 3) $[16,17]$. Marker localization was based on the Calibrated Anatomical Systems Technique (CAST). Tracking markers consisted of left and right anterior superior iliac spine, middle of the sacroiliac joint, two cluster markers (eight markers) on the lateral side of the femur and lower leg, posterior prominence of the calcaneus, distal end of second and fifth metatarsal bones [16].

After recording motions with 6 calibrated digital JVC camcorders (JVC Inc., Japan) (filming frequency of 200 frames per second), the researchers extracted threedimensional (3D) characteristics of markers by SIMI ${ }^{\circledR}$ Motion Analysis workstation software (SIMI Reality Motion Systems GmbH, Unterschleissheim, Germany).

A

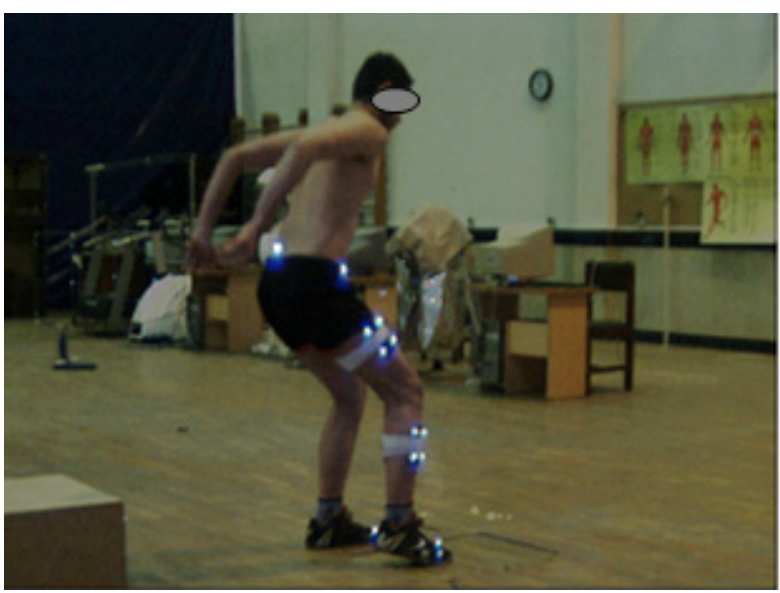

Sagittal view

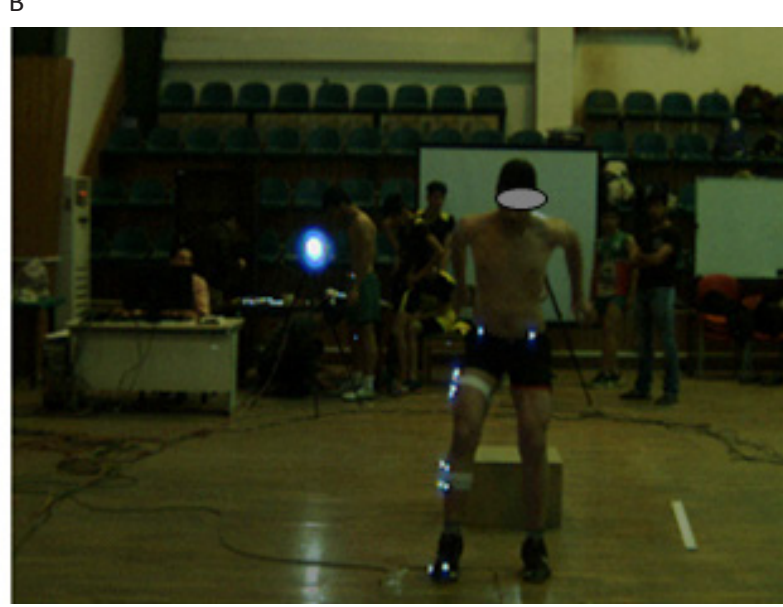

Frontal view

The researchers imported and processed all data using MATLAB (The MathWorks, Inc., Natick, MA, USA). Each subject's data sequence was time normalized to $0 \%-100 \%$ of the landing duration. They used motion data to create joint angle data sequences. The knee joint angles of flexion-extension, abduction-adduction, and internal rotation-external rotation were estimated and averaged over 3 trials. They also calculated 3D knee joint angles and expressed it in the reference frame of the proximal segment. The local coordinate system was placed at the proximal endpoint of the joint. The frontal plane was defined by the orientation of the $\mathrm{x}$-axis (flexion and extension).

The z-axis (internal and external rotation) passed through each segment's proximal and distal endpoints. They oriented the $y$-axis (abduction and adduction) orthogonal to both the $\mathrm{x}$-axis and $\mathrm{z}$-axis. For each trial, knee frontal and transverse plane angles were obtained at the time of peak and average

B

Figure 3. Marker set during jump-landing maneuver 
knee flexion angles. They normalized all variables to the participant's weight $(\mathrm{kg})$.

The researchers also created a database in Microsoft Excel 2010 for each participant that contained the three knee angles. Using these data, they analyzed movement in sagittal, frontal, axial and transverse planes during landing and their correlations with clinical data.

\section{Data analysis}

Researchers used SPSS V. 21 for statistical analysis of the data. The Pearson correlation test was performed to examine the correlation between core muscle endurance and 3D knee angles during jump-landing $(\mathrm{P} \leq 0.05)$.

\section{Results}

The results are presented in Tables 2 and 3. The relationship between the kinematic variables of the knee during jump-landing and the clinical parameters of the core muscles' endurance is presented in Table 4. A statistically significant correlation was demonstrated between the peak and average value of knee flexion angle and right and left side bridge tests. Moreover, a significant negative correlation was observed between knee internal rotation and trunk flexors' endurance test value. Other findings for the kinematic of the knee showed no significant relationship with the clinical parameters of the core muscles' endurance.

\section{Discussion}

The best screening method for lower extremity injury risk in military personnel is still unknown, although there is increasing evidence in an athletic population that decreased knee flexion and increased abduction during landing from a jump may contribute to increased ACL injury risk [18]. Moreover, the factors associated with dangerous knee kinematics among the military have not been identified. The study investigated the correlation between trunk muscle endurance and knee kinematics during jump-landing in military personnel. The finding suggests that the endurance of quadratus lumborum and obliques muscles in both sides, which have been assessed through side bridge test, was positively correlated with peak and mean knee flexion angles during landing.

This means that a better test result for the side bridge test is associated with more flexion angle of the knee during landing, which is a characteristic of a "soft" and appropriate pattern. The pattern proved to correlate with less injury risk. In contrast, the more "upright" and erect landing shows one of the risk factors of ACL injury [19].

Table 2. Core endurance test (McGill protocol)

\begin{tabular}{ccc}
\hline Test & Mean \pm SD & Range \\
\hline Prone plank $[\mathrm{s}]$ & $95.15 \pm 27.15$ & $44-155$ \\
\hline Right side bridge $[\mathrm{s}]$ & $61.85 \pm 29.91$ & $20-145$ \\
\hline Left side bridge $[\mathrm{s}]$ & $63.70 \pm 28.23$ & $29-128$ \\
\hline Extensor endurance $[\mathrm{s}]$ & $100.60 \pm 31.69$ & $54-178$ \\
\hline Flexor endurance $[\mathrm{s}]$ & $106.50 \pm 44.47$ & $61-204$ \\
\hline
\end{tabular}

$J M R$

Table 3. Jump-landing kinematic

\begin{tabular}{ccccc}
\hline Joint Position & Peakb & Range & Averagec \\
\hline Knee-flexion angle $\left[^{\circ}\right]$ & 72.05 & $40.98-88.45$ & 31.54 & $10.74-48.89$ \\
Knee-abduction angle $\left[^{\circ}\right]$ & -7.72 & $-39.46-82.08$ & -0.35 & $-13.28-22.11$ \\
Knee-internal rotation angle $\left[^{\circ}\right]$ & -24.12 & $-83.23-89.04$ & -15.25 & $-53.28-12.58$ \\
\hline
\end{tabular}

A. Positive values indicate flexion, adduction, and external-rotation directions; B. Indicates the peak over the entire stance phase (range: $0 \%-100 \%$ ); C. Indicates an average over the entire stance phase (range: $0 \%-100 \%$ ). 
Table 4. The Pearson correlation coefficients (r) between clinical measurements of core muscles' endurance and kinematic data of the knee joint during Jump-landing phase

\begin{tabular}{|c|c|c|c|c|c|c|c|}
\hline Variables & & Flexion [Average] & $\begin{array}{l}\text { Flexion } \\
\text { [Peak] }\end{array}$ & $\begin{array}{l}\text { Abduction } \\
\text { [Average] }\end{array}$ & $\begin{array}{l}\text { Abduction } \\
\text { [Peak] }\end{array}$ & $\begin{array}{c}\text { Rotation [Aver- } \\
\text { age] }\end{array}$ & $\begin{array}{c}\text { Rotation } \\
\text { [Peak] }\end{array}$ \\
\hline \multirow{2}{*}{ Prone plank } & r & -0.093 & 0.217 & -0.011 & 0.162 & 0.187 & 0.069 \\
\hline & $P$ & 0.696 & 0.357 & 0.963 & 0.495 & 0.429 & 0.774 \\
\hline \multirow{2}{*}{ Right side bridge } & r & $0.511 *$ & $0.505^{*}$ & 0.192 & 0.089 & -0.324 & -0.364 \\
\hline & $P$ & 0.021 & 0.023 & 0.416 & 0.710 & 0.164 & 0.114 \\
\hline \multirow{2}{*}{ Left side bridge } & r & $0.486^{*}$ & $0.445^{*}$ & 0.211 & 0.087 & -0.279 & -0.374 \\
\hline & $P$ & 0.030 & 0.049 & 0.371 & 0.715 & 0.233 & 0.104 \\
\hline \multirow{2}{*}{$\begin{array}{l}\text { Extensor endur- } \\
\text { ance }\end{array}$} & r & -0.019 & 0.190 & -0.008 & -0.075 & 0.024 & -0.023 \\
\hline & $P$ & 0.936 & 0.422 & 0.973 & 0.752 & 0.922 & 0.924 \\
\hline \multirow{2}{*}{ Flexor endurance } & $r$ & 0.326 & 0.109 & -0.135 & -0.326 & $-0.496 *$ & -0.325 \\
\hline & $\mathrm{P}$ & 0.161 & 0.648 & 0.570 & 0.161 & 0.026 & 0.162 \\
\hline
\end{tabular}

*Indicates statistical significance $\mathrm{P} \leq 0.05$.

This finding is compatible with Leetun et al. and Hodges et al., who confirmed the role of abdominal muscles in lower extremity kinematics and injury risk [20, 21]. Leetun and colleagues used a combination of core endurance/strength measures in males and females in a prospective study. They tried to correlate these measures and athletes at risk for injuries in the lower extremities. The authors found that stronger hip abduction and mainly external rotation were related to a decreased risk of lower extremity injury [20]. Moreover, Hodges and coworkers in an electromyography study examined the roles of trunk muscles and the prime movers for hip flexion, abduction, and extension during hip movements in each direction. The researchers indicated that the transversus abdominis and oblique abdominal muscles were associated with the function regardless of the direction of the forces [21].

The transverse and internal oblique abdominal muscles and the sacrotuberous ligament have connections to the deep layer of the thoracolumbar fascia [22]. In weakness, there is a tendency for the local stabilizing muscles of the pelvis to fatigue. Fatigue of these stabilizing muscles creates inefficiencies in movements and consequently increases the workload of the lower limb musculature. The increased workload will predispose to early muscle fatigue and subsequent injury [23]. The quadratus lumborum is also reported to be an essential stabilizer of the lumbar spine, because of its structural characteristics and location [12, 24]. It is active for most tasks that require lumbar flexion and extension moment production [20].

The hip musculature acts synergistically with quadratus lumborum to stabilize the trunk over the lower extremities and transfer force from the lower limbs to the pelvis and spine [25]. In a single leg weight-bearing position, the ground reaction force vector is shown to be located medial to the hip joint creating an external abduction moment at the hip joint. Activation of the lateral core muscles creates an opposing internal moment, neutralizing excessive adduction of the femur [26]. Studies of associated kinematic changes occurring with poor core stability support a biomechanical model of femoral adduction and internal rotation with valgus knee positioning [27-29].

Moreover, the abdominal muscles' endurance (flexor endurance test) had a negative correlation with mean knee internal rotation during landing. Thus, any decrease in abdominals' endurance may probably increase the internal rotation of the knee dynamically. Chuter et al. reported that decreased knee flexion in conjunction with tibial internal rotation was correlated with direct impingement of the ACL on the intercondylar notch, increasing the risk of injury [30]. Yet, the authors found no study considering the relationship between abdominal muscle endurance and dynamic knee rotation in jumplanding. The abdominals have also been reported to stabilize the spine through co-contraction with the lumbar extensors [31].

Researchers further suggest that the abdominals also control excessive anterior pelvic tilt, which is believed to be coupled with femoral internal rotation and adduction [32]. In his review, Devlin suggested that abdominal weakness and fatigue may be a contributing factor to hamstring injuries [23]. Zazulak et al, in their prospective study, showed that greater trunk displacement after sudden force release was correlated with knee, ligament, 
and ACL injuries in injured athletes, especially in females [11].

Contrary to other studies, the researchers could not find a significant correlation between knee abduction (valgus) and trunk muscle endurance. The authors conducted McGill test with subjects in supine, prone, and sitting positions and the assessment technique may be different in inferring core stabilizing ability in the standing position used during the double-leg jump-landing task. Likewise, one of the reasons making the present results different from other studies may lay in the fact that the majority of researchers focus on female subjects due to their greater risk of ACL injury rather than their male counterparts. Furthermore, it is essential to consider that movement control is multifactorial and influenced by many components such as muscle elasticity, nervous system, anatomical structure, besides endurance, and they may affect these injurious movements [33].

This investigation had some limitations. The participation of male Iranian military personnel with the age range of 20-37 years may influence the generalizability of the results to the general population. Further, the male militaries that took part in this research had been recruited from the general population, but they were assessed before basic military training. Thus, the results of the study should be considered regarding the specificity of our sample. Moreover, core muscle strength measure was not evaluated in the article with a dynamometer, and the trunk endurance time test (McGill protocol) was used for assessing the ability to recruit core musculature. Therefore, the differences between task methodologies may result in different outcomes.

The study indicated a positive correlation between side bridge test result and peak/mean knee flexion angles during landing. Moreover, a significant negative correlation was found between abdominal muscles' endurance (flexor endurance test) and mean knee internal rotation. The trunk muscles are important stabilizers of the knee joint during activity [34]. A growing body of evidence indicates a correlation between lower limbs injury with neuromuscular dysfunction of the trunk $[35,36]$. Timed tests of trunk muscle endurance such as McGill protocol are more accessible, but it is unclear whether endurance is merely enough for improving knee stability and decreasing the risk of injury. The results suggest that increasing trunk muscle endurance might help control the knee during landing from a jump.

The results have significant implications for males who may be at risk for lower extremity injury during dynamic activities. Prospective studies, however, are needed to clarify the relationship between trunk muscle endurance and lower limb injury rate. The researchers also recommend future research with more variables (trunk muscle EMG, etc.) and test protocols that are more dynamic and sport-specific.

\section{Ethical Considerations}

\section{Compliance with ethical guidelines}

All ethical principles were considered in this article. The participants were informed about the purpose of the research and its implementation stages; they were also assured about the confidentiality of their information; Moreover, They were allowed to leave the study whenever they wish, and if desired, the results of the research would be available to them.

\section{Funding}

This research did not receive any specific grant from funding agencies in the public, commercial, or not-forprofit sectors.

\section{Authors contributions}

All authors contributed in preparing all parts of the research.

\section{Conflict of interest}

The authors declared no conflict of interest.

\section{Acknowledgements}

Authors would like to express their gratitude to heads of the Sports Sciences Department of the University of Mazandaran for their permission to use the laboratory.

\section{References}

[1] Willson JD, Dougherty CP, Ireland ML, Davis IM. Core stability and its relationship to lower extremity function and injury. Journal of the American Academy of Orthopaedic Surgeons . 2005; 13(5):316-25. [DOI:10.5435/00124635-20050900000005] [PMID]

[2] Jamison ST, McNeilan RJ, Young GS, Givens DL, Best TM, Chaudhari AM. Randomized controlled trial of the effects of a trunk stabilization program on trunk control and knee loading. Medicine \& Science in Sports \& Exercise. 2012 44(10):1924-34. [DOI:10.1249/MSS.0b013e31825a2f61] [PMID]

[3] Bouisset S. Relationship between postural support and intentional movement: Biomechanical approach. Archives of 
International Physiology, Biochemistry and Biophysics. 1991; 99(5):77-92. [DOI:10.3109/13813459109145919] [PMID]

[4] Bliven K, Anderson B. Core stability training for injury prevention. Sports Health. 2013; 5(6):514-22 [DOI:10.1177/1941738113481200] [PMID] [PMCID]

[5] Pfile KR, Hart JM, Herman DC, Hertel J, Kerrigan DC, Ingersoll CD. Different exercise training interventions and drop landing biomechanics in high school female athletes. Journal of Athletic Training. 2013; 48(4):450-62. [DOI:10.4085/1062 6050-48.4.06] [PMID] [PMCID]

[6] Shirey M, Hurlbutt M, Johansen N, King GW, Wilkinson SG, Hoover DL. The influence of core musculature engagement on hip and knee kinematics in women during a single leg squat. International Journal of Sports Physical Therapy. 2012; 7(1):1-12. [DOI:10.1249/01.MSS.0000401382.61642.9c]

[7] Hickey L, Kline P, Ireland M, Noehren, B. Hip and trunk muscle dysfunction: Implications for anterior cruciate ligament injury prevention. Annals of Joint. 2017; 2(5):1-8 [DOI:10.21037/aoj.2017.05.07]

[8] Borghuis J, Hof AL, Lemmink KA. The importance of sensory-motor control in providing core stability: Implications for measurement and training. Sports Medicine. 2008; 38(11):893916. [DOI:10.2165/00007256-200838110-00002] [PMID]

[9] Kibler WB, Press J, Sciascia A. The role of core stability in athletic function. Sports Medicine. 2006; 36(3):189-98. [DOI:10.2165/00007256-200636030-00001] [PMID]

[10] Zazulak BT, Hewett TE, Reeves NP, Goldberg B, Cholewicki J. The effects of core proprioception on knee injury: A prospective biomechanical-epidemiological study. American Journal of Sports Medicine. 2007; 35(3):368-73. [DOI:10.1177/0363546506297909] [PMID]

[11] Zazulak BT, Hewett TE, Reeves NP, Goldberg B, Cholewicki J. Deficits in neuromuscular control of the trunk predict knee injury risk: A prospective biomechanical-epidemiologic study. American Journal of Sports Medicine. 2007; 35(7):1123 30. [DOI:10.1177/0363546507301585] [PMID]

[12] McGill SM, Childs A, Liebenson C. Endurance times for low back stabilization exercises: Clinical targets for testing and training from a normal database. Archives of Physical Medicine and Rehabilitation. 1999; 80 (8):941-4. [DOI:10.1016/ S0003-9993(99)90087-4]

[13] Evans K, Refshauge KM, Adams R. Trunk muscle endurance tests: Reliability, and gender differences in athletes. Journal of Science and Medicine in Sport. 2007; 10(6):447-55 [DOI:10.1016/j.jsams.2006.09.003] [PMID]

[14] Moreau C, Green B, Johnson C, Moreau S. Isometric back extension endurance tests: A review of the literature. Journal of Manipulative and Physiological Therapeutics. 2001; 24(2):110-22. [DOI:10.1067/mmt.2001.112563] [PMID]

[15] Padua DA, Marshall SW, Boling MC, Thigpen CA, Garrett WE Jr, Beutler AI. The Landing Error Scoring System (LESS) is a valid and reliable clinical assessment tool of jump-landing biomechanics: The JUMP-ACL study. American Journal of Sports Medicine. 2009; 37(10):1996-2002 [DOI:10.1177/0363546509343200] [PMID]

[16] Andriacchi TP, Alexander EJ, Toney MK, Dyrby C, Sum J. A point cluster method for in vivo motion analysis: Applied to a study of knee kinematics. Journal of Biomechanical Engi neering. 1998; 120(6):743-9. [DOI:10.1115/1.2834888] [PMID]

[17] Camomilla V, Cereatti A, Vannozzi G, Cappozzo A. An optimized protocol for hip joint centre determination using the functional method. Journal of Biomechanics. 2006; 39(6):1096106. [DOI:10.1016/j.jbiomech.2005.02.008] [PMID]

[18] Nakagawa TH, Petersen RS. Relationship of hip and ankle range of motion, trunk muscle endurance with knee valgus and dynamic balance in males. Physical Therapy in Sport. 2018; 34:174-9. [DOI:10.1016/j.ptsp.2018.10.006] [PMID]

[19] Decker MJ, Torry MR, Wyland DJ, Sterett WI, Richard Steadman J. Gender differences in lower extremity kinematics, kinetics and energy absorption during landing. Clinica Biomechanics (Bristol, Avon). 2003; 18(7):662-9. [DOI:10.1016/ S0268-0033(03)00090-1]

[20] Leetun DT, Ireland ML, Willson JD, Ballantyne BT, Davis IM. Core stability measures as risk factors for lower extremity injury in athletes. Medicine \& Science in Sports \& Exercise. 2004; 36(6):926-34. [DOI:10.1249/01.MSS.0000128145.75199. C3] [PMID]

[21] Hodges PW, Richardson CA. Contraction of the abdominal muscles associated with movement of the lower limb. Physical Therapy. 1997; 77(2):132-42. [DOI:10.1093/ptj/77.2.132] [PMID]

[22] Pool-Goudzwaard AL, Vleeming A, Stoeckart R, Snijders CJ, Mens JM. Insufficient lumbopelvic stability: A clinical, anatomical and biomechanical approach to 'a-specific' low back pain. Manual Therapy. 1998; 3(1):12-20. [DOI:10.1054/ math.1998.0311] [PMID]

[23] Devlin L. Recurrent posterior thigh symptoms detrimental to performance in Rugby Union. Sports Medicine. 2000; 29(4):273-87. [DOI:10.2165/00007256-200029040-00005] [PMID]

[24] Cholewicki J, McGill SM. Mechanical stability of the in vivo lumbar spine: Implications for injury and chronic low back pain. Clinical Biomechanics. 1996; 11(1):1-15. [DOI:10.1016/0268-0033(95)00035-6]

[25] Akuthota V, Nadler SF. Core strengthening. Archives of Physical Medicine and Rehabilitation. 2004; 85(1):86-92. [DOI:10.1053/j.apmr.2003.12.005] [PMID]

[26] Willson JD, Dougherty CP, Ireland ML, McClay Davis I. Core stability and its relationship to lower extremity function. The Journal of the American Academy of Orthopaedic Surgeons. 2005; 13(5):316-25. [DOI:10.5435/00124635-20050900000005] [PMID]

[27] Earl JE, Hoch AZ. A proximal strengthening program improves pain, function, and biomechanics in women with patellofemoral pain syndrome. American Journal of Sports Medicine. 2011; 39(1):154-63. [DOI:10.1177/0363546510379967] [PMID]

[28] Ferber R, Noehren B, Hamill J, Davis IM. Competitive runners with a history of iliotibial band syndrome demonstrate atypical hip and knee kinematics. Journal of Orthopaedic \& Sports Physical Therapy. 2010; 40(2):52-8. [DOI:10.2519/ jospt.2010.3028] [PMID]

[29] Pollard CD, Sigward SM, Powers CM. Limited hip and knee flexion during landing is associated with increased frontal plane motion and moments. Clinical Biomechan- 
ics (Bristol Avon). 2010; 25(2):142-6. [DOI:10.1016/j.clinbioe mech.2009.10.005] [PMID] [PMCID]

[30] Chuter VH, Janse de Jonge XA. Proximal and distal contributions to lower extremity injury: A review of the literature. Gait Posture. 2012; 36(1):7-15. [DOI:10.1016/j.gaiti post.2012.02.001] [PMID]

[31] Bergmark A. Stability of the lumbar spine: A study in mechanical engineering. Acta Orthopaedica Scandinavica. 1989; 230:1-54. [DOI:10.3109/17453678909154177] [PMID]

[32] Ireland ML. The female ACL: Why is it more prone to injury? Orthopedic Clinics of North America. 2002; 33(4):637-51. [DOI:10.1016/S0030-5898(02)00028-7]

[33] Martinez AF, Lessi GC, Carvalho C, Serrao FV. Association of hip and trunk strength with three-dimensional trunk, hip and knee kinematics during a single-leg drop vertical jump. The Journal of Strength and Conditioning Research. 2018; 32(7):1902-8. [DOI:10.1519/JSC.0000000000002564] [PMID]

[34] Willson JD, Ireland ML, Davis I. Core strength and lower extremity alignment during single leg squats. Medicine \& Science in Sports \& Exercise. 2006; 38(5):945-52. [DOI:10.1249/01. mss.0000218140.05074.fa] [PMID]

[35] De Blaiser C, Roosen P, Willems T, Danneels L, Bossche LV, De Ridder R. Is core stability a risk factor for lower extremity injuries in an athletic population? A systematic review. Physical Therapy in Sport. 2018; 30:48-56. [DOI:10.1016/j. ptsp.2017.08.076] [PMID]

[36] Scattone Silva R, Nakagawa TH, Ferreira AL, Garcia LC, Santos JE, Serrão FV. Lower limb strength and flexibility in athletes with and without patellar tendinopathy. Physical Therapy in Sport. 2016; 20:19-25. [DOI:10.1016/j. ptsp.2015.12.001] [PMID] 
This Page Intentionally Left Blank 